\title{
Patient-Specific Analysis of Myocardial Strains in Left Bundle Branch Block Based on Computational Models
}

\author{
Kimi P Owashi ${ }^{1}$, Elena Galli ${ }^{1}$, Arnaud Hubert ${ }^{1}$, Erwan Donal ${ }^{1}$, Alfredo I Hernández ${ }^{1}$, Virginie Le \\ Rolle $^{1}$ \\ ${ }^{1}$ Univ Rennes, CHU Rennes, Inserm, LTSI - UMR 1099, F-35000 Rennes, France
}

\begin{abstract}
This paper proposes a patient-specific model-based estimation of myocardial strain signals and the evaluation of echo-based parameters, adapted to patients with left bundle branch block (LBBB). The left ventricle ( $L V)$ was divided into 16 segments in order to evaluate concurrently different regions at the ventricular contraction process. For each LV segment, some parameters, associated with the active and passive components of the cardiac muscle, the electro-mechanical driving function and the electrical depolarization time, were identified using evolutionnary algorithms. The proposed approach was evaluated on data obtained from 3 LBBB patients. From patientspecific simulations, we also analysed electrical activation delay and myofiber contractility in LV segments. A close match was observed between experimental and simulated myocardial strain curves for all the subjects. The root mean square error (RMSE) is equal to $2.87( \pm 1.00)$, $2.49( \pm 0.55)$ and $3.63( \pm 0.81)$ for the anterior ischemic, the lateral ischemic and the non-ischemic $L B B B$ patients, respectively. The proposed patient-specific model-based approach may be a useful tool for understanding LV mechanical dyssynchrony and identifying patients suitable for cardiac resynchronization therapy (CRT).
\end{abstract}

\section{Introduction}

While normal left ventricular (LV) contraction is rapid and synchronous, left bundle branch block (LBBB) induces abnormal asynchronous contraction patterns, which could impairs the efficiency of ventricular contraction. These abnormalities in LV performance could be compensated by cardiac resynchronization therapy (CRT). However, approximately $30 \%$ of patients undergoing CRT according to current guidelines [1] show little or no improvement [2]. Although, previous studies have suggested that the analysis of mechanical dyssynchrony by speckle tracking echocardiography (STE) can improve prediction of response to CRT [3], guidelines still neglect the value of the assessment of LV mechanical dyssynchrony for the prediction of CRT-response.

The interpretation of features extracted from echocardiography could be difficult due the complexity of mechanisms involved in the generation of ventricular contraction (mechano-hydraulic interactions, electrical activation and propagation, etc.) and the multidimensionality of the problem. In this context, computational modelling could help to improve the interpretability of longitudinal strain curves, as it allows the integration of physiological knowledge into data processing [4].

In this paper, we aimed at proposing a patient-specific model-based approach of the cardiovascular system (CVS) in order to assist the analysis and improve the interpretability of myocardial strains and echo-based parameters. A novel integrated model of the CVS was proposed and parameters were identified in order to provide patientspecific model-based strain curves of 3 LBBB patients. Ischemic and non-ischemic patients were selected because patients with non-ischemic cardiomyopathy have shown a better response to CRT compared with ischemic LBBB patients [5]. Finally, contractility parameters and electrical activation delays in the different LV regions were evaluated for each patient.

\section{Methods}

\subsection{Clinical data}

We prospectively included 3 CRT-candidates with LBBB: 2 patients with ischemic heart disease and anterior or lateral myocardial scar, and 1 patient without ischemic cardiomyopathy. The study was carried out in accordance with the principles outlined in the Declaration of Helsinki on research in human subjects and received specific ethical approval from the local Medical Ethical Committee. All patients signed a written informed consent before the participation to the study protocol. The experimental dataset includes the ECG and the measured regional myocardial strain curves obtained by the STE at transthoracic echocardiography in apical 4-chamber, 2-chamber and 3- chamber 


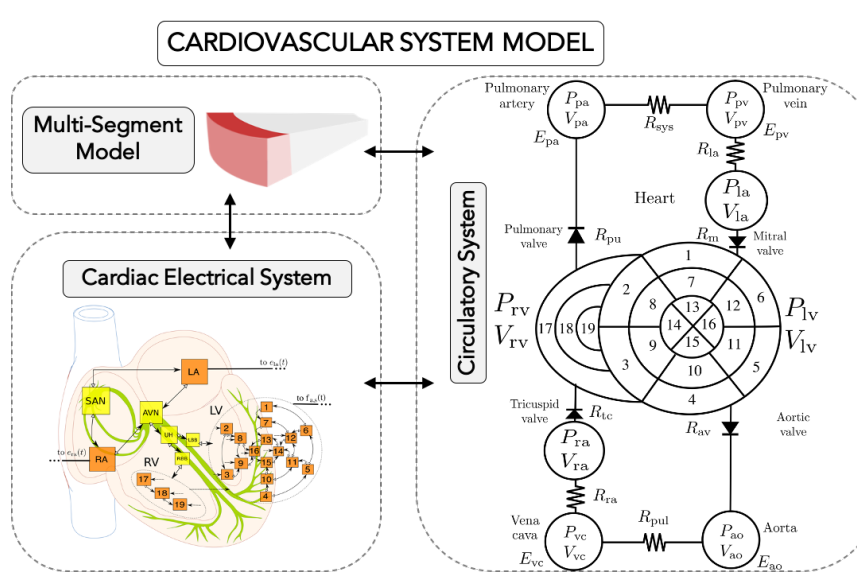

Figure 1. Integrated model of the CVS proposed, based on the coupling of the electrical, mechanical and hydraulic activity of the heart.

views. For the 2 patients with ischemic cardiomyopathy the location of the scar was made at cardiac magnetic resonance imaging (MRI) by the assessment of the distribution of late gadolimium enhancement and then confirmed by echocardiography.

\subsection{Model description:}

Cardiac electrical system: A set of interconnected cellular automata, adapted from [4, 6], represents the cardiac electrical activity of the model. Each automaton represents different cardiac regions that cycle between four electrical activation states: slow diastolic depolarization (SDD), upstroke depolarization period (UDP), absolute refractory period (ARP) and relative refractory period (RRP).

The whole model consists of 26 automata representing: the sinoatrial node (NSA), right and left atria (RA and LA), the atrioventricular node (NAV), upper bundle of His (UH), bundle branches (RBB and LBB), 3 segments of right ventricle (RV) and 16 segments of left ventricle (LV).

Right and left atrium: Atrial pressure $\left(P_{a}\right)$ is calculated using a linear relationship of the volume $\left(V_{a}\right)$ and the time-variant elastance $\left(E_{a}\right)$.

$$
\begin{aligned}
P_{a}\left(V_{a}, t\right) & =E_{a}\left(V_{a}(t)-V_{a, d}\right) \\
E_{a}(t) & =e_{a}(t)\left(E_{a, \text { max }}-E_{a, \min }\right)+E_{a, \min }
\end{aligned}
$$

The $E_{a}$ is controlled by a Gaussian driving function $\left(e_{a}\right)$ that represents contraction and relaxation of the atria.

$$
e_{a}(t)=A \cdot \exp \left(-B \cdot\left(t_{a}-C\right)^{2}\right)
$$

where $t_{a}$ is the time elapsed since the atrial activation by the automata corresponding to the right and left atriums.
Parameters $A, B$ and $C$ are used to control the rise and peak of the atrial systole.

Multi-segments right and left ventricles: Each LV and RV automaton triggers an electro-mechanical driving function (EMDF), which represents in a simplified manner, the complex processes involved in the electro-mechanical coupling at the tissue-level [7]:

$$
f_{v, s}(t)=\left[\frac{\left(\frac{t}{\alpha_{1} T}\right)^{n_{1}}}{1+\left(\frac{t}{\alpha_{1} T}\right)^{n_{1}}}\right] \cdot\left[\frac{1}{1+\left(\frac{t}{\alpha_{2} T}\right)^{n_{2}}}\right] \cdot A_{\max }
$$

The first and second terms in Eq. 3 represent ventricle segment contraction and relaxation presented after an electrical activation, respectively. $T$ is the heart period, $\alpha_{1}, \alpha_{2}$ are shape parameters, and $n_{1}, n_{2}$ control the steepness of the curve. $A_{\max }$ is the maximum EMDF value and $s \in$ $\left\{S_{l v}, S_{r v}\right\}$ with $S_{l v}=\{$ BasAnt, BasAntSep, BasInfSep, BasInf, BasInfLat, BasAntLat, MidAnt, MidAntSep, MidInfSep, MidInf, MidInfLat, MidAntLat, ApAnt, ApSep, ApInf, ApLat $\}$ and $S_{r v}=\{$ BasRV, MidRV, ApRV\}.

Concerning each segment $s$, cardiac mechanical activity can be separated into active and passive components:

$T_{s}=T_{\text {pass }, s}+T_{a c t, s}$

Passive [8] and active [9] myocardial tension in each segment are represented by Eq. 4 and Eq. 5, respectively:

$$
\begin{aligned}
T_{\text {pass }, s} & =K_{\text {pass }} \cdot\left(36 \cdot \max \left(0, l / l_{\text {ref }}-0.1\right)^{2}\right. \\
& \left.+0.1\left(l / l_{\text {ref }}-0.1\right)+0.0025 \exp \left(10 \cdot l / l_{\text {ref }}\right)\right)
\end{aligned}
$$

where $K_{\text {pass }}$ is a a gain that could be related to passive stiffness, $l$ and $l_{\text {ref }}$ are current and reference sarcomere lengths.

$$
T_{a c t, s}=K_{a c t} \cdot\left(1+\beta\left(l / l_{r e f}-1\right)\right) \cdot \frac{f_{v, s}^{2}}{f_{v, s}^{2}+f_{c}^{2}}
$$

where $K_{a c t}$ is a gain that could be related to myofiber contractility, $\beta$ and $f_{c}$ are constants related with the muscle kinetic. Myocardial strain, $\varepsilon_{s}^{\text {model }}$, is calculated based on the length variation in each segment.

By the Laplace's law we established the relationship between the pressure $P_{s}$ and the tension $T_{s}$ in each segment.

Finally, ventricular flow $Q_{v}$ is calculated taking into account the flow contribution of each one of the segments $Q_{s}$ and the intraventricular cavity flow $Q_{c}$ :

$Q_{v}(t)=Q_{c}(t)+\sum_{s} Q_{s}(t)$

Segments, associated with the septum, are treated separately since their pressure depends of the pressure gradient across the septal wall: $P_{\text {sept }}=P_{l v}-P_{r v}$

Systemic and pulmonary circulations: Concerning the circulatory models [10], pressures on vessel or cardiac chambers are calculated as a linear pressure-volume relationship, similar to Eq. 1.

The pressure gradient across chambers and the corresponding vascular resistance are then used to calculate flows as: $Q=\Delta P / R$. 

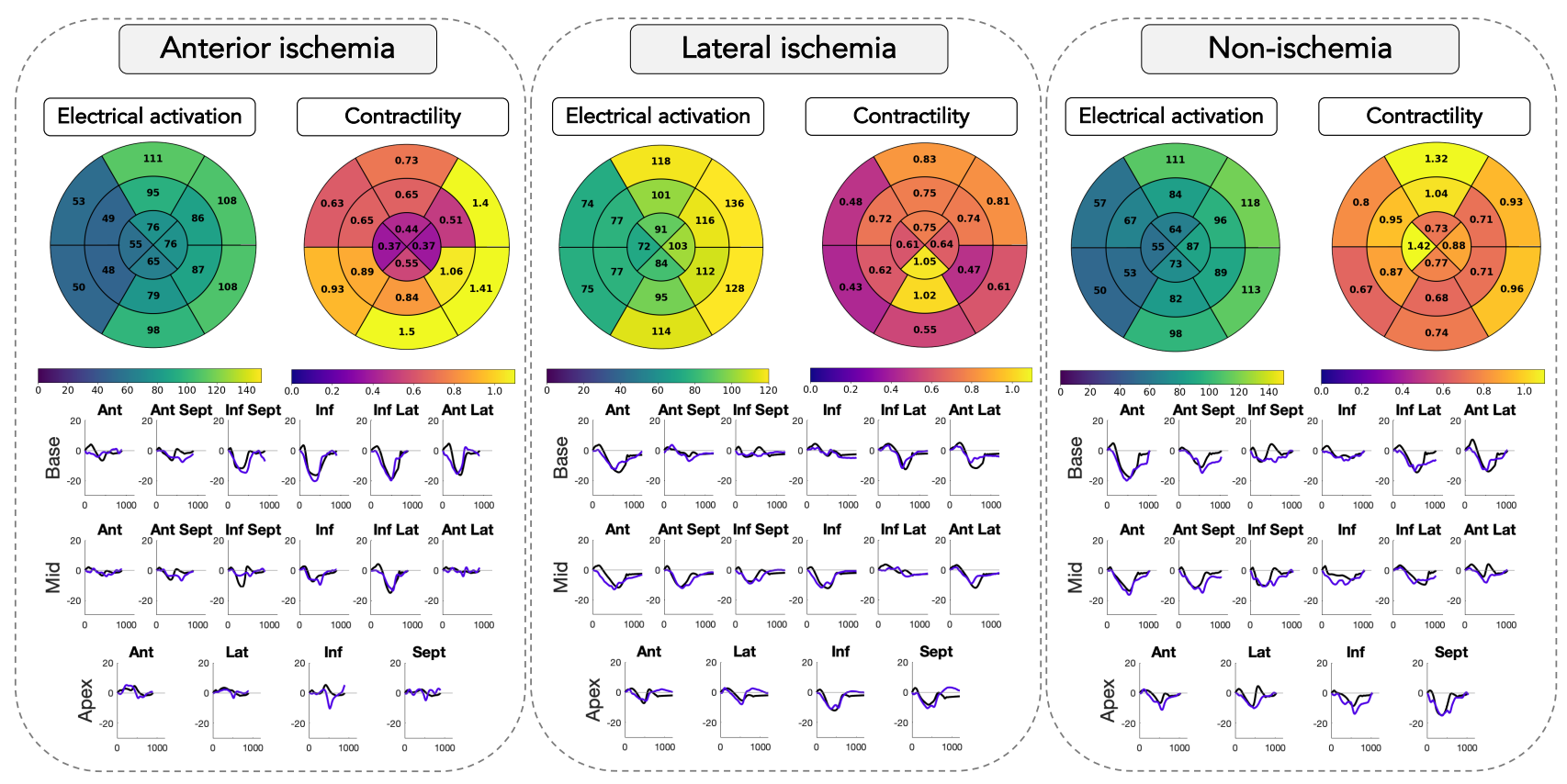

Figure 2. Myocardial strain signals obtained from clinical data (blue) and patient-specific simulations (black). Bull's-eye representations of the segmental activation delay (left) and contractility (right) for the anterior ischemic patient (left), the lateral ischemic patient (middle) and the non-ischemic patient (right).

The volume change of each cardiac and vessel chamber is computed from the integral of their respective net flow: $\Delta V(t)=\int\left(Q_{\text {in }}-Q_{\text {out }}\right) \mathrm{dt}$.

\subsection{Parameter identification}

For each LV segment, some parameters, associated with the active $\left(K_{a c t}\right)$ and passive $\left(K_{\text {pass }}\right)$ components of the cardiac muscle, the $\operatorname{EMDF}\left(n_{1}, n_{2}, \alpha_{1}, \alpha_{2}\right)$ and the electrical depolarization time (UDP), were identified using evolutionnary algorithms. The identification strategy, seeks to find the best set of parameters that minimizes the error function $J$ between experimental and simulated signals for each segment:

$$
J_{s}=\frac{1}{T_{c}} \sum_{t_{e}=0}^{T_{c}-1}\left|\varepsilon_{s}^{e x p}\left(t_{e}\right)-\varepsilon_{s}^{\text {model }}\left(t_{e}\right)\right|+\alpha\left|\varepsilon_{s, \text { min }}^{e x p}-\varepsilon_{s, \text { min }}^{\text {model }}\right|
$$

where $\varepsilon_{s}^{e x p}$ and $\varepsilon_{s}^{\text {model }}$ are the myocardial strain signals obtained from available clinical data and simulated outputs, respectively. $t_{e}$ corresponds to the time elapsed since the onset of the identification period and $T_{c}$ is the duration of a cardiac cycle. $J_{s}$ represents the error function for each segment, therefore, $J=\sum_{s=1}^{16} J_{s}$.

From patient-specific simulations, identified UDP and $K_{\text {act }}$ parameters of each LV segment were used to analyse segmental electrical activation delay and myofiber contractility, respectively. Bull's-eye representation were plotted in order to compare these parameters in the 3 LBBB patients.

\section{Results}

Figure 2 compares the simulated and experimental LV regional myocardial strain curves for the anterior ischemic, the lateral ischemic and the non-ischemic LBBB patients. The results reflects a satisfying adaptation of the model to different strain morphologies, since a close match was observed between experimental and simulated data for all the cases. The root mean square error (RMSE) is equal to $2.87( \pm 1.00), 2.49( \pm 0.55)$ and $3.63( \pm 0.81)$ for the anterior ischemic, the lateral ischemic and the non-ischemic LBBB patients, respectively.

From the model-based approach, electrical activation delay and regional contractility were estimated and represented by bulls-eye plots (Fig. 2) in the 3 LBBB cases. Ischemic patients present reduced contractility in anterior and lateral segments, corresponding with the areas of ischemia reported in the patients. Regional contractility results allow ischemic and non-ischemic cases distinction, where reduced contractility could be associated with damaged tissues. Electrical activation bulls-eye representation shows an important electrical activation delay between the lateral and the septal wall of the LV in all the cases. 


\section{Discussion}

The main contributions of this work are the propositions of 1) a novel CVS model that integrates a multi-segment representation of the ventricles and 2) parameter identification procedure able to reproduce patient-specific myocardial strain signals showing satisfying morphologies.

Although finite element models were widely used in the literature to represent cardiac electrical and mechanical activities [11], these models are characterized by a significant number of parameters, require relevant computational resources and, consequentially, their ability to be identified is more complex. The proposed model is based on a functional integration of interacting physiological processes, by taking into account: $i$ ) the electro-mechanical coupling, $i$ i) the interactions between the myocardial wall and the blood inside the cavity and iii) a simplified representation of systemic and pulmonary circulation and $i v$ ) interventricular interaction. This allows the representation of the main cardiac properties required to tackle the problem under study, like the Frank-Starling law and the influence of preload and afterload. Moreover, the model resolution has been adapted to the problem, keeping a similar abstraction level as clinicians for the analysis of strain signals.

Patient-specific parameters were obtained for $3 \mathrm{LBBB}$ patient. The mean error between observed and synthesized strain signals, after parameter identification, remains particularly low. From the model-based approach, the electrical activation delay shows consistent results with the physiological processes presented in LBBB patients. The localization of ischaemic segments have shown to be coherent with the clinical analysis based on the location of the scar by MRI and confirmed by echocardiography.

Clinically, reproducing LV deformation signals of the patient may be useful for the interpretation of the electromechanical data, for the understanding of the biological substrate (ventricular) as the basis of LV dysfunction and, may be in the future, for the planning of CRT procedures.

\section{Conclusion}

Assessment of myocardial strain signals and the evaluation of certain echo-based parameters by patient-specific simulations based on computational models may be a useful tool for understanding LV mechanical dyssynchrony and identifying patients suitable for cardiac resynchronization therapy (CRT). Future works will be dedicated to more extensive evaluations including a greater population of patients.

\section{Acknowledgments}

This work was supported by the French National Research Agency (ANR) (ANR-16-CE19-0008-01) (project
MAESTRo) and the French Brittany council (ADvICE project).

\section{References}

[1] Brignole M, Auricchio, et al. 2013 ESC Guidelines on cardiac pacing and cardiac resynchronization therapy. The task force on cardiac pacing and resynchronization therapy of the European Society of Cardiology (ESC). EP Europace August 2013;15(8):1070-1118. ISSN 1099-5129.

[2] Zareba W, Klein H, et al. Effectiveness of cardiac resynchronization therapy by QRS morphology in the multicenter automatic defibrillator implantation trial-cardiac resynchronization therapy (MADIT-CRT). Circulation March 2011;123(10):1061-1072. ISSN 1524-4539.

[3] Risum N, Tayal B, et al. Identification of typical left bundle branch block contraction by strain echocardiography is additive to electrocardiography in prediction of long-term outcome after cardiac resynchronization therapy. Journal of the American College of Cardiology August 2015;66(6):631641. ISSN 1558-3597.

[4] Le Rolle V, Hernàndez A, et al. Model-based analysis of myocardial strain data acquired by tissue doppler imaging. Artificial Intelligence in Medicine 2008;44:201-19.

[5] Yokoshiki H, Mitsuyama H, et al. Cardiac resynchronization therapy in ischemic and non-ischemic cardiomyopathy. Journal of Arrhythmia October 2017;33(5):410-416. ISSN 1880-4276.

[6] Hernández AI, Carrault G, Mora F, Bardou A. Modelbased interpretation of cardiac beats by evolutionary algorithms: signal and model interaction. Artificial Intelligence in Medicine 2002;26(3):211-235.

[7] Stergiopulos N, et al. Determinants of stroke volume and systolic and diastolic aortic pressure. Am J Physiol 1996; 270:H2050-H2059.

[8] Lumens J, Delhaas T, Kirn B, Arts T. Three-wall segment (TriSeg) model describing mechanics and hemodynamics of ventricular interaction ;37(11):2234-2255. ISSN 15739686.

[9] Hunter PJ, McCulloch AD, ter Keurs HE. Modelling the mechanical properties of cardiac muscle ;69:289-331. ISSN 0079-6107.

[10] Ojeda D, Le Rolle V, et al. Sensitivity analysis and parameter estimation of a coronary circulation model for triplevessel disease. IEEE Transactions on Biomedical Engineering 2014;61(4):1208-1219.

[11] Le Rolle V, Galli E, Danan D, et al. Sensitivity analysis of a left ventricle model in the context of intraventricular dyssynchrony. Acta Biotheoretica March 2020;68(1):4559. ISSN 1572-8358.

Address for correspondence:

Kimi P. Owashi

LTSI-Université de Rennes 1. Campus de Beaulieu. Bât 22. 35042. Rennes. France

kimi-piedad.owashi@univ-rennes1.fr 